\title{
La inserción organizacional en la economía ${ }^{1}$
}

Andrés Zambrano*

\begin{abstract}
Resumen
Formalidad e informalidad como sectores en la economía han sido definidas en la estadística nacional desde diversos puntos de vista: desde la empresa, a partir del número de personas que ocupa; desde el ocupado, si trabaja por cuenta propia siendo o no profesional; desde la ocupación, según las condiciones de contratación. Si bien los conceptos pueden estar claros, al momento de estudiar actividades económicas los límites entre ambos sectores son difusos. A partir de una encuesta a 1.166 microempresas en 19 ciudades de Venezuela, se ha definido la formalidad e informalidad a partir de las relaciones organizacionales que se establecen con el Estado y con el mercado, componiendo un orden de tres tipos de informalidad.
\end{abstract}

Palabras claves: Economía - formalidad - informalidad.

\begin{abstract}
Formal and informal sector in the economy have been defined in national statistics from different points of view: from the business, based on the number of employees; from the worker, if he is self-employed, being or not professional; from the work, by the contract condition. Although the concepts may be clear, when studying economic activities the borderline between the two sectors is fuzzy. A total 1,166 small businesses in 19 cities of Venezuela were surveyed. From the organizational relations established with the government and the market we obtained the definition of the formal and informal sector, in which we found an order of three types of informality.
\end{abstract}

Keywords: Economy - formality - informality.

1 Los estudios realizados en el Centro de Investigación Social (CISOR), el año 2007, El microseguro en Venezuela. Una exploración en el sector microempresarial; y el año 2011, AUGE el programa de Apoyo a Unidades de Gestión Económica del Centro al Servicio de la Acción Popular CESAP, han desembocado en la escritura de este artículo. Agradezco al personal de CISOR que contribuyó en el trabajo de recopilación de los datos en los estudios anteriormente nombrados y que han sido utilizados en este artículo. Gracias a Alberto Gruson por la orientación conceptual y a Fernando Blanco y Blas Regnault por sus observaciones al texto.

* Sociólogo, Investigador del Centro de Investigación Social (CISOR), Caracas, Venezuela. Correo electrónico: andresviana@gmail.com 


\section{PREÁMBULO CONCEPTUAL}

Las personas, agrupándose o de manera individual, pueden tener el interés de organizar la práctica de algún deporte, un arte, un culto religioso o una actividad comercial. Este trabajo de establecer un interés legítimo pasa en un primer momento por considerar las buenas razones por las que se debe fundar una iniciativa, es decir, instituir los fines de una organización. Este trabajo fundacional, que se inicia con cierto ánimo utópico, se va concretando en la medida que se ordenan y movilizan recursos, capacidades y herramientas, se practican técnicas y, en los casos más elaborados, hasta se protocolizan procesos, consiguiendo que las finalidades se hagan operativas. Entonces, se ha constituido una organización que está lista para procurar una inserción social en un cierto contexto, exponiendo al público un interés, sea prestar un servicio (lucrativo o no), sea producir un bien, sea asociar a otras organizaciones.

Toda organización tiene esa doble faceta, la primera es la institución, son los fines para los que se establece la actividad organizada, el proyecto o propósito específico que orienta el conjunto de elementos disponibles; es esto último la otra cara de la moneda, es decir, los recursos, su arreglo metódico y coherente para la realización de determinados trabajos, es la vertiente instrumental de la actividad que hace eficaz la voluntad planteada en el proyecto (Chantal y Seguin, 1987). Entonces, la organización se define como "un conjunto de medios gobernados por una autoridad en vista de asegurar una función reconocida como legítima en una sociedad dada" (Touraine, 1993: 252)2. Toda organización está compuesta por tres dimensiones, cada una definida por pares opuestos que generan tensiones durante su funcionamiento. Se espera que la organización no funcione solo en alguno de los extremos del par, sino más bien que mantenga en equilibrio esta tensión operando entre los extremos. Los desequilibrios atentan contra el funcionamiento de la organización y sólo cierta armonía permiten una acción eficaz en la colectividad. Los tres equilibrios fundamentales que debe procurar la organización son ${ }^{3}$ (Touraine, 1993, 1978):

1. El dinamismo que es consecuencia de la tensión existente entre una orientación hacia la consecución de las exigencias provenientes del ambiente (los objetivos externos) y, por otro lado, hacia los arreglos y asuntos de la dinámica de los socios y del personal (las exigencias internas). Los desequilibrios se presentan al funcionar solamente en alguno de los dos extremos, es decir, la mera conquista de los objetivos a costa de los miembros, o sacrificar los fines para satisfacer solamente las necesidades del personal.

2. La capacidad que resulta de los ajustes entre atender los aspectos económicos de la organización, sus procedimientos y operatividad (la técnica) y la capacidad de conducción de estos recursos (el poder), siendo que los recursos se resisten al poder que se aplica sobre ellos, de allí la tensión. Los desequilibrios se presentan cuando las decisiones son acertadas,

2 Entre las primeras obras del sociólogo francés Alain Touraine se presenta un planteamiento teórico que, si bien comprende los elementos burocráticos de la organización, trata principalmente sobre el aspecto organizacional de la sociedad. Léase: Production de la société (1973), Le livre de poche (1993), Introducción a la sociología (1978).

3 Los elementos que constituyen el par de una dimensión han sido colocados entre paréntesis. 
pero no se tienen las habilidades necesarias para la ejecución; o, por el contrario, cuando la organización depende solamente de oportunidades técnicas casuales que sirven para gestionar sus recursos sin lograr ejercer una verdadera capacidad de decisión.

3. La inserción que es el resultado de oponer, por un lado, la condición de la organización como centro de decisión en el que es libre para definir sus normas de funcionamiento interno y sus intercambios con el ambiente (la autonomía) y, por otro lado, la subordinación de la organización frente al orden social que condiciona y regula su acción, sus fines y sus operaciones (la dependencia). Los desequilibrios se dan cuando la dinámica interna no encuentra vínculos organizacionales con el entorno o, por el contrario, toda la acción está subordinada y mediatizada por regulaciones externas, por tanto la organización no tiene centro de decisión.

En la dinámica concreta de la colectividad, la autonomía y la dependencia pueden evidenciarse en una regulación dada únicamente por el mercado (total autonomía), opuesta a una regulación solamente del poder público (total dependencia) (Gruson, 2011). Esta oposición crea un marco para que la organización tenga una inserción en un determinado lugar en la colectividad.

En este último equilibrio se encuentra la definición sociológica que fundamenta las consideraciones que se harán sobre la inserción organizacional en la economía a lo largo de este escrito, en el que se pretende ilustrar empíricamente el concepto de inserción definida desde la idea de normación de las relaciones entre organizaciones, sea con normas explicitadas formalmente o solo con regularidades informales, es decir, desde el punto de vista de si los intercambios entre actividades organizacionales son burocráticos o no. Para ello, se inicia con un planteamiento desde la estadística nacional que clasifica las actividades socioproductivas en formales e informales desde una variedad de perspectivas.

\section{PLANTEAMIENTO}

En las estadísticas utilizadas para el estudio de la ocupación y de las actividades socioproductivas, tanto la formalidad como la informalidad han sido consideradas desde el punto de vista de la empresa, de la ocupación y del ocupado. En ocasiones desde una combinación de algunos de estos conceptos con la finalidad de una cuantificación propia del sistema de registros de la nación, información fundamental para evaluar y diseñar políticas públicas referidas al empleo.

En Venezuela, el Sistema Estadístico Nacional define a los ocupados en el sector informal de la economía como el conjunto de patronos, empleados y trabajadores familiares que laboran en empresas con menos de cinco personas ocupadas, también los trabajadores por cuenta propia no profesionales, incluyendo las personas que trabajan como servicio doméstico (OCEl, 1997: 167). Aquí son dos categorías las que distinguen una inserción formal de una informal. La primera es una cualidad de la empresa: su tamaño apreciado desde el número de ocupados. La segunda categoría es una cualidad del ocupado, compuesta por la combinación de (1) la categoría de ocupación de la persona, en este caso un trabajador 
por cuenta propia, quien "no depende de un patrono, ni tiene empleados u obreros a su cargo y ofrece sus servicios en forma individual y privada" (OCEl, 1997: 171), y (2) el nivel educacional alcanzado por el ocupado, siendo que la distinción es profesional/no profesional. Si bien estas categorías son claras, no lo es el concepto de informalidad: "piénsese en un joyero que trabaja con dos o tres asistentes (pertenecen al sector informal), o en un profesional convertido en taxista de cuenta propia (que, por profesional, pertenece al sector formal)" ${ }^{\prime 4}$.

La informalidad también se define como una cualidad de la ocupación ejercida por el trabajador, haciendo referencia a la estabilidad de la contratación, la protección social, el tipo de remuneración y los beneficios laborales ${ }^{5}$. La regularidad del empleo, es decir, que este sea asalariado y con contrato estable, que los empleadores sean responsables por las cargas fiscales y las contribuciones a la seguridad social, y que la relación laboral se rija por mecanismos institucionales de contratación, termina siendo un conjunto de categorías que identifica lo precario de la ocupación. Sin embargo, el trabajo precarizado no tiene que ser indicador de informalidad, se puede estar empleado en una condición vulnerable en una empresa que cumple criterios de formalidad, sea porque es la condición que ofreció el empleador o porque el empleado así lo prefirió, por ejemplo, quienes no desean tener ataduras contractuales para así trabajar en más de un establecimiento; en ambos casos es lo que en ocasiones se conoce como una contratación de forma flexible, que puede llegar al punto de no tener un contrato registrado (práctica vista tanto en pequeñas, medianas y grandes empresas). Por lo tanto, asimilar al trabajador asalariado en estas condiciones a un ocupado en el sector informal pareciera ser un manejo erróneo, ya que se trata más bien de la calidad de la ocupación de la persona; más aún si se ha evidenciado que en el sector formal de la economía las relaciones laborales pueden ser más o menos flexibles. Estas prácticas de trabajo (vinculadas a su precarización o flexibilización) son, en todo caso, una dinámica gerencial de las organizaciones. Posiblemente, el aumento de su práctica no tiene que repercutir en un crecimiento del sector informal de la economía.

Vinculado a lo anterior, también se utiliza la acepción "trabajo decente" para hacer referencia a la calidad de las condiciones de la ocupación, pero en este caso desde el punto de vista del derecho de los trabajadores a la libertad de asociación y discusión con empresarios y autoridades gubernamentales, la no discriminación laboral, la ausencia de trabajo forzado e infantil en condiciones abusivas, la seguridad o protección social, y las condiciones físicas en las actividades y de los establecimientos laborales, entre otras consideraciones ${ }^{6}$. Desde este punto de vista la finalidad es fomentar y reivindicar algunos derechos fundamentales para generar condiciones laborales cada vez más dignas; por lo tanto, parece que no se apunta a definir un sector en la economía.

4 Véase CISOR (2005: 3), trabajo en el que se menciona esta condición de la estadística nacional de ocupación en Venezuela, en el marco de un estudio sobre la microempresa.

5 Para una comparación del tratamiento estadístico de la ocupación en el mundo, véase Organización Internacional del Trabajo (1992). Una versión revisada y actualizada de la segunda edición publicada en 1992, en la que se considera una extensa lista de países (incluyendo Venezuela), se encuentra disponible en: http://laborsta.ilo. org/applv8/data/SSMs.html

6 Puede leerse sobre el tema en Organización Internacional del Trabajo (2002). Un uso de este concepto se encuentra en Feijo y De Souza (2009). 
Si bien los sectores formal e informal se han definido a partir de una cualidad administrativa de la empresa, una característica del ocupado y una condición de la ocupación, la pretensión es verificar empíricamente el concepto sociológico de inserción descrito anteriormente. Para ello se ha considerado una serie de transacciones que las actividades pueden tener tanto con el Estado como con el mercado. Es de esperar que en estos dos planos se establezcan algunas regulaciones en el intercambio, el primero, como aparato administrativo, al normar las transacciones en la colectividad a través de un sistema legal y burocrático; y el segundo, desde el sector privado mediante organizaciones comerciales y gremiales, al regular la dinámica de competencia y beneficio en la oferta de bienes y servicios. Estos sectores definen formas tanto fiscales como institucionales con la pretensión de estructurar un sistema para el intercambio. Sin embargo, entre los intercambios totalmente regulados de manera explícita y los intercambios que se dan en total ausencia de regulación formal, el esquema se complejiza en la medida en que, entre ambos extremos, se materializan transacciones caracterizadas por formas difusas.

El proceso de regular los intercambios, sea con normas explicitadas formalmente o sólo con regularidades informales, distingue cualidades en las transacciones, en las maneras de iniciarlas y mantenerlas en la colectividad. Desde este punto de vista, no todas las actividades se insertan de igual forma y, a su vez, un cúmulo de cierto tipo de relaciones crea contextos de inserciones diferentes para el intercambio. Una tipología que ayude al estudio de la inserción de las actividades en la economía, desde el punto de vista de sus relaciones organizacionales, es lo que se presentará en las siguientes páginas.

Para lo anterior, y usando como fuente de dato actividades microempresariales, se han tomado las reglas prevenidas formalmente en términos de Estado y mercado dominante para desde ellas describir las transacciones mercantiles de organizaciones y así componer contextos de inserción. La elaboración resultante se denomina "contextos de inserción organizacional". Elaborar esta tipología implicó trabajar con datos provenientes del encuestamiento a 1.166 dueños de microempresas ubicadas en 19 centros poblados de Venezuela. En la primera parte del escrito se describe la fuente de información, los parámetros con que fue constituida la muestra y el tipo de procesamiento al que se sometieron los datos. Posteriormente se describe la tipología de inserción elaborada; esta se propone como una variable y se ilustra presentando algunos cuadros de frecuencia con datos del encuestamiento a dueños de microempresas. Finalmente, se hacen algunas reflexiones en torno a la variable inserción como estructura normativa para la previsibilidad de los intercambios.

\section{DISEÑO DE LA MUESTRA}

En el año 2007 el Centro de Investigación Social (CISOR) Ilevó a cabo un encuestamiento a dueños de microempresas ${ }^{7}$. En el año 2010 se hizo un segundo encuestamiento que sirvió

\footnotetext{
7 Contratación hecha por la empresa Münchener de Venezuela, con el interés de indagar la posibilidad de insertar
} en el sector microempresarial un servicio de aseguramiento. Los resultados están en Zambrano (2007). 
para complementar y corregir asuntos tratados en el trabajo de campo del año $2007^{8}$. En ambos encuestamientos el objeto de estudio fue la microempresa. Para esto se constituyó una muestra considerandos tres parámetros: primero, el tamaño del negocio según el número de personas ocupadas en este; segundo, la rama de actividad económica según la Clasificación Industrial Internacional Uniforme (CIIU); y tercero, una clasificación de los centros poblados del país según su desarrollo urbano. A continuación se detalla la manera en que se utilizaron los criterios para el diseño de la muestra.

\subsection{El tamaño de la empresa}

La microempresa se encuentra en un conjunto heterogéneo de unidades económicas de pequeña escala. Estas unidades comprenden desde estrategias unipersonales de empleo de sectores de la población en precariedad económica, hasta empresas con niveles importantes de inversión y productividad, pasando por unidades de producción generadas a partir de la desconcentración de los procesos fabriles de determinadas ramas económicas de la mediana y gran industria. Esta afirmación da una noción del sector de la economía al que se hace referencia, pero es aún una idea difusa. De allí que sea necesario establecer una suerte de pivote que ofrezca una definición parametrizada útil para el estudio de las actividades de esta escala.

Una fuente de información para una aproximación al mundo de la microempresa es la Encuesta de Hogares por Muestreo (EHM), orientada al monitoreo de la situación del empleo y de la ocupación, que se realiza en Venezuela en forma continua desde 1967 por el Instituto Nacional de Estadística (INE) (antes OCEI: Oficina Central de Estadística e Informática), proporcionando resultados oficiales semestrales. Mediante las preguntas relativas al establecimiento comercial en el que se desempeñan los ocupados, la encuesta permite identificar un primer parámetro susceptible de ser comparado a escala internacional: el número de personas ocupadas en el negocio. Estos son considerados (actualmente) en tres rangos por la EHM, siendo: menos de 5 personas; de 5 a 20 personas; más de 20 personas. Los rangos de tamaño de empresa permiten establecer el primer parámetro para la definición de la microempresa. Esta se distribuye en tres tamaños, que son: empresa unipersonal; empresa de 2 a 4 personas, y empresa de 5 a 20 ocupados (OCEI, 1995; INE, 2006).

En la empresa unipersonal se encuentra el trabajador por cuenta propia, profesional y no profesional. El rango de 2 a 4 personas hace límite con la definición del sistema estadístico nacional del sector informal, constituido por aquellas actividades económicas que comprenden hasta 4 ocupados, exceptuando al profesional por cuenta propia que forma parte del sector formal. Todas las actividades con 5 o más ocupados forman parte del

8 Trabajo hecho gracias al apoyo financiero del Fondo Multilateral de Inversiones FOMIN (perteneciente al Banco Interamericano de Desarrollo BID) que financió la evaluación del programa de créditos a microempresarios del Grupo Social CESAP. Los resultados de este trabajo están plasmados en Zambrano y Moncrieff (2011). 
sector formal de la economía. El límite de 20 ocupados se considera para la definición de pequeña o microempresa.

Siendo el número de ocupados un primer criterio de obtención de información útil para delimitar el mundo microempresarial, también deben considerarse los tipos de actividad económica llevados a cabo en este sector.

\subsection{Las ramas de actividad económica}

Un segundo parámetro susceptible a la comparación internacional es la actividad económica a la que se dedica la empresa, constituyendo una variable que ha sido objeto de clasificaciones internacionales minuciosas. La División de Estadísticas de las Naciones Unidas propuso en 1948 una Clasificación Industrial Internacional Uniforme (CIIU), la misma ha sido actualizada en varias oportunidades, siendo que la empleada por la EHM es parecida a la versión de 1968.

Para empresas con hasta 20 ocupados, la EHM permite distinguir 33 ramas de actividad económica en las que se ejerce actividad, es decir, son ramas que corresponden a la esfera microempresarial en Venezuela. Motivado a que algunas registran una proporción de ocupados muy baja respecto del total, en el encuestamiento del año 2007 se optó solamente por 17 de las 33 ramas de actividad. En la Tabla 1 se listan ${ }^{9}$.

En las 17 ramas de actividad económica listadas se distribuye el 95\% de las personas ocupadas en el sector microempresarial en Venezuela (CISOR, 2005) ${ }^{10}$. Cada rama de actividad comprende una diversidad de casos o tipos de negocios. En cada uno de los centros poblados seleccionados para la muestra se encuestaron casos para estas ramas de actividad, con la excepción de agricultura, pesca y comercio mayorista, actividades que se encuentran solamente en ciertos centros poblados dependiendo de su tamaño y ubicación geográfica.

\subsection{Los centros poblados}

El diseño de la muestra fue hecho a partir de un método de selección de centros poblados basado en la estructura urbano-regional de Venezuela; esta es una variable compuesta en CISOR que refleja la dinámica socioeconómica del país combinando el grado de urbanización

9 Las ramas de actividad económica en las que las microempresas se desenvuelven han sido tomadas de los resultados de un estudio de exploración metodológica cuya finalidad es ilustrar como la EHM brinda una información que bien podría explotarse para caracterizar el mundo microempresarial, a pesar de que se trata de una encuesta orientada al monitoreo de la situación del empleo y la ocupación en Venezuela. A manera de marco general, el estudio presenta resultados sobre el empleo en Venezuela para los años 1995 y 2000 ; luego hace foco en las empresas de pequeña escala, disponiendo al lector una clasificación tipológica de las microempresas y una caracterización de las diferentes clases. Véase: Centro de Investigación Social (2005).

10 Cálculos válidos para los años 1995 y 2000. Procesamiento de CISOR de la Encuesta de Hogares, primer semestre 1995 y 2000. Comprende empresas unipersonales (trabajadores por cuenta propia). 
TABLA 1

Ramas de actividad del sector microempresarial.

Venezuela, años 1995 y 2000

\begin{tabular}{|l|l|}
\hline CÓDIGO CIIU & \multicolumn{1}{|c|}{ RAMA DE ACTIVIDAD ECONÓMICA } \\
\hline 111 & Agricultura y cría \\
\hline 130 & Pesca \\
\hline 311 & Fabricación de productos alimentarios \\
\hline $321,322,324$ & Fabricación de textiles, prendas de vestir y calzado \\
\hline 331,332 & Fabricación con madera; carpintería \\
\hline 341,342 & Fabricación con papel; imprentas \\
\hline 381 & Fabricación de productos metálicos \\
\hline 500 & Construcción \\
\hline 610 & Comercio mayorista \\
\hline 620 & Comercio minorista \\
\hline 631 & Restaurantes; expendios de comida y bebida \\
\hline 711 & Transporte terrestre \\
\hline 831 & Bienes inmuebles \\
\hline 832,833 & Servicios a empresas; alquiler de equipos \\
\hline 933 & Servicios médicos, odontológicos, veterinarios de reparación, talleres mecánicos \\
\hline 951 & \\
\hline 959 &
\end{tabular}

Fuente: CISOR (2005). Datos del INE, Encuesta de hogares, primer semestre 1995 y 2000. Procesamiento directo CISOR, no oficial.

y el tamaño poblacional de los centros poblados, además de su cercanía a la ciudad capital. La variable es una categorización representativa del desarrollo del país que busca evitar el estudio sustentado en la división político-administrativa de las entidades federales, que trae como inconveniente la no consideración de la heterogeneidad interna de la entidad, producto del desarrollo desigual. La estructura urbano-regional permite ser usada como un 
criterio para el establecimiento de muestras que abarquen la diversidad de la población nacional (Gruson, 2008; García et al., 2009).

La estructura urbano-regional puede presentarse en 6 categorías (o ámbitos) en la que se distribuye la población de Venezuela, estas son: (U1) el área de la Capital de país -Caracas-; (U2) las ciudades mayores de la región central con sus ciudades satélites, y los satélites de Caracas; (U3) las ciudades grandes del país; (U4) casi todas las ciudades medianas; (U5) algunas ciudades medianas, las ciudades pequeñas y demás mayores de 2.500 habitantes; (U6) la población dispersa en poblados rurales, exceptuando los del centro del país ${ }^{11}$. Siguiendo esta clasificación los centros poblados considerados en el encuestamiento fueron:

- Ciudades Capital-centro (U1 y U2): Caracas; Maracay, Valencia, Los Teques.

- Ciudades grandes-medianas del interior (U3 y U4): Barcelona-Puerto La Cruz, Ciudad Guayana, Maracaibo, Barquisimeto; Ciudad Bolívar, San Juan de los Morros, Valera, San Cristóbal, Mérida.

- Poblados pequeños y rurales (U5 y U6): Caripe, Ortiz-Parapara de Ortiz, Tucacas; San Agustín (Municipio Caripe. Edo. Monagas), El Toco (Municipio Juan German Roscio. Edo. Guárico), Jajó (Municipio Urdaneta. Edo. Trujillo).

Estos centros poblados se distribuyen en el Distrito Capital y 13 entidades federales (de las 24 que conforman el país), a saber: Anzoátegui, Aragua, Bolívar, Carabobo, Falcón, Guárico, Lara, Mérida, Miranda, Monagas, Táchira, Trujillo y Zulia. Selección que considera las 6 regiones geográficas en que se divide Venezuela: Centro-norte costera; Llanera; Occidental; Andina; Oriental; y Guayana.

\section{RECOLECCIÓN DE DATOS}

Con los tres parámetros anteriores se compuso un marco muestral a partir de la combinación de tres tamaños de microempresa con 17 ramas de actividad, resultando 51 casos a encuestar en cada uno de los centros poblados de los 6 ámbitos urbano-regionales. La combinación de estos tres parámetros permite considerar las distintas actividades microempresariales y sus posibles tamaños en una diversidad de centros urbanos (grandes y medianos), las pequeñas ciudades del interior y los poblados rurales. Así, la representatividad más que cuantitativa es cualitativa, es decir, busca considerar las diferentes cualidades que forman el universo de estudio; por esto, el procedimiento fundamental para establecer la muestra fue abarcar la totalidad de los criterios establecidos en las consideraciones técnicas en la medida en que se distribuye el encuestamiento.

11 Ciudades mayores: 400 mil y más habitantes; Ciudades grandes: 95 mil a 400 mil habitantes; Ciudades medianas: 45 mil a 95 mil habitantes; Ciudades pequeñas: 2500 a 45 mil habitantes; Poblados rurales: menos de 2500 habitantes. 
Tanto en el estudio del año 2007 como del año 2010 se distribuyeron las encuestas a manera de cuotas en los casos resultantes de cruzar los criterios técnicos ya explicados. En el año 2007 se encuestaron 857 dueños de microempresa, en el año 2010 fueron 309; totalizando 1.166 empresarios encuestados. A continuación se detalla la distribución resultante.

TABLA 2

Distribución de los microempresarios encuestados por ámbito urbano-regional y rama de actividad, 2007 y 2010 (valores absolutos)

\begin{tabular}{|c|c|c|c|c|}
\hline \multirow[b]{2}{*}{ RAMA DE ACTIVIDAD } & \multicolumn{3}{|c|}{ CIUDADES DEL ÁMBITO } & \multirow[b]{2}{*}{ TOTALES } \\
\hline & $\begin{array}{l}\text { CAPITAL-CENTRO } \\
\text { DEL PAÍS }\end{array}$ & $\begin{array}{l}\text { GRANDES-MEDIANAS } \\
\text { DEL INTERIOR }\end{array}$ & $\begin{array}{l}\text { POBLADOS } \\
\text { RURALESY } \\
\text { DISPERSOS }\end{array}$ & \\
\hline Agricultura y pesca & 0 & 0 & 32 & 32 \\
\hline Fabricación de alimentos & 29 & 25 & 11 & 65 \\
\hline Fabricación textil-calzado & 35 & 25 & 9 & 69 \\
\hline Fabricación con madera & 27 & 17 & 9 & 53 \\
\hline Fabricación con papel & 22 & 10 & 1 & 33 \\
\hline Fabricación con metal & 29 & 22 & 6 & 57 \\
\hline Construcción civil & 27 & 15 & 11 & 53 \\
\hline Comercio mayorista & 19 & 9 & 2 & 30 \\
\hline Comercio minorista & 115 & 140 & 21 & 276 \\
\hline Comida y bebida preparada & 49 & 36 & 16 & 101 \\
\hline Transporte urbano & 57 & 52 & 10 & 119 \\
\hline Servicios a empresas & 37 & 22 & 5 & 64 \\
\hline Servicios médicos & 17 & 13 & 3 & 33 \\
\hline Servicios de reparación & 47 & 26 & 13 & 86 \\
\hline Servicios personales & 41 & 40 & 14 & 95 \\
\hline Totales & 551 & 452 & 163 & 1.166 \\
\hline
\end{tabular}

Fuente: Bases de datos de microempresas 2007 y 2010, elaboradas en CISOR. 
Véase que un agricultor en centros urbanos, un negocio mayorista o algunas actividades de fabricación y de servicios en un poblado rural, son casos prácticamente inexistentes. El criterio numérico acerca del total de encuestas a realizar en cada ámbito no guarda relación con la población que cada uno alberga; en su lugar, se optó por un criterio que dé cuenta de la diversidad de negocios que el ámbito presenta en cada rama de actividad económica.

\section{LA INSERCIÓN ORGANIZACIONAL: PROCESAMIENTO DE DATOS Y RESULTADOS}

Se han considerado las transacciones mercantiles a partir de las reglas prevenidas formalmente por el Estado y el mercado para la fundación y posterior funcionamiento de la organización, esto con la finalidad de observar contextos de intercambios, desde los más hasta los menos regulados formalmente. Estas reglas se listan a continuación ${ }^{12}$ :

Relación con el Estado:

- Registro mercantil.

- Registro fiscal en el sistema tributario nacional y en el municipal.

- Permiso de habitabilidad del establecimiento o de tránsito del vehículo.

- Permisología (específica para ciertas actividades comerciales).

Relación con mercados (insumos, servicios y clientes):

- Factura legal del insumo o servicio recibido por un proveedor.

- Factura legal del producto o servicio vendido a un cliente.

Relación con la actividad misma:

- Contabilidad.

- De haber empleados:

* Registro de horario de trabajo, libro de vacaciones y horas extras.

* Registro en la seguridad social, ahorro habitacional, paro forzoso.

* Cálculo de beneficios salariales (prestaciones, utilidades).

En los cuestionarios se colocaron estos requisitos formales a manera de preguntas, posteriormente transformadas en variables dicotómicas para distinguir la presencia o ausencia de un requisito. Los datos de las 1.166 encuestas realizadas a dueños de microempresas se dispusieron en el programa SPAD (Système Portable pour l'Analyse de Données). El

12 Los requisitos han sido tomados de la sección sobre trámites oficiales del Estado venezolano vigentes (y consultados) para el año 2010 y están disponibles al público en el sitio www.gobiernoenlinea.gob.ve (Ejecutivo nacional, 2010). 
método para el procesamiento de los datos se ha hecho con estadística descriptiva y análisis factorial multivariable y de conglomerado. Los dos últimos cálculos estadísticos son útiles para la formación de agrupaciones homogéneas a lo interno y heterogéneas en relación con otros grupos. Este procedimiento permitió dar un orden a las relaciones establecidas por la actividad con el Estado y con el mercado.

Distintas pruebas hechas con análisis de correspondencia múltiple resultaron en el descarte de variables por el hecho de no presentar correlaciones significativas, lo que permitió reducir la dimensionalidad del estudio; luego, la clasificación automática de los conglomerados resultantes (representados en un dendograma) permitió elaborar una clasificación de 8 conglomerados que terminaron ordenándose en 4, lo que conformó grupos más homogéneos a lo interno y diferentes entre sí. Como resultante las variables seleccionadas son: 1. Actividad registrada (si/no); 2. Declara/cancela algún impuesto (si/no); 3. Recibe factura de su proveedor (si/no $)^{13}$.

Las variables antes indicadas son las que mejor componen un marco de transacciones definidas por lo regulado/no regulado, desde el punto de vista del Estado y del mercado. Aquí, el registro de la actividad, la fiscalización impositiva y la evidencia de una operación comercial mediante la recepción de una factura, definen contextos de inserción. El orden generado permite diferenciar una inserción propiamente organizacional de las actividades en la economía a partir del estudio de actividades de pequeña y muy pequeña escala. Léanse a continuación las cuatro inserciones:

La inserción formal: es aquella donde las transacciones se ejercen a través de regulaciones formales y explícitas. Respecto del Estado la actividad es fiscalizable, por tanto se declara una carga impositiva y se está en un registro administrativo. Adicionalmente, ante el mercado los intercambios son declarables, es decir, la transacción consta en papeles con vigencia legal (facturas).

La inserción sancionable: está caracterizada por el incumpliendo de las normas del Estado, sin embargo, la actividad se inserta en la economía dominante, donde el intercambio es regulado mediante la facturación; por tanto, la formalidad responde más a requisitos del mercado que a controles del Estado. Si el aparato de fiscalización ejerce el dominio necesario, el evasor podría ser sancionado ${ }^{14}$.

13 Recibir una factura legal de un proveedor es un indicador de formalidad en la relación comercial, ya que permite hacer el intercambio comercial regulable (no se oculta la transacción y se puede enjuiciar la calidad de la prestación). La bondad de este indicador, en contraste con entregar una factura al cliente, se evidencia al considerar el transporte de pasajeros, donde la empresa que presta el servicio no entrega una factura al usuario (bien pudiendo pertenecer al sector formal de la economía), pero sí la puede recibir del proveedor.

14 Téngase en cuenta que en ningún momento se hace referencia en el texto a transacciones de bienes ilegales o actividades violatorias de los derechos de ciertas categorías de población, como el trabajo infantil en condiciones precarias. En este caso (como en otros de inserción no formal) lo sancionable es la forma en la que se comercia un bien o servicio y no el bien en sí mismo. 
La inserción marginal: se da en los márgenes con la economía dominante. La factura legal que evidencia una operación no se vincula con esta forma de inserción. Sin embargo, el Estado reconoce la actividad, ya que se cumplen normas gubernamentales al registrar el negocio y/o cancelar algún impuesto. A su vez puede ser que haya tolerancia o desconocimiento por el Estado de las relaciones con clientes o proveedores que pertenecen a la economía de subsistencia ${ }^{15}$.

La inserción eventual: aquí las transacciones carecen de toda regulación formal, la actividad solamente conecta de manera precaria y casual con un mercado de regulaciones explícitas. No hay fiscalización por parte del Estado y el intercambio económico no se controla en término de garantías. Lo esperado es que sea poca la cantidad de actividades que se establecen en una inserción eventual, que en ocasiones es casi nula ${ }^{16}$.

Este orden se distribuye gráficamente en una combinación de regulación Estadomercado. A efectos de la literatura existente, las inserciones sancionable, marginal y eventual pueden agruparse en lo que comúnmente se denomina informalidad ${ }^{17}$, entonces, además de la formal, se distinguen tres tipos o modalidades de inserción informal en la dinámica económica.

TABLA 3

Contextos de inserción organizacional

\begin{tabular}{|l|l|c|c|}
\hline \multirow{2}{*}{\multicolumn{2}{|c|}{}} & \multicolumn{2}{c|}{ REGULACIÓN POR EL MERCADO } \\
\cline { 2 - 4 } & $\begin{array}{c}\text { ECONOMÍA } \\
\text { DOMINANTE }\end{array}$ & $\begin{array}{c}\text { ECONOMÍA DE } \\
\text { SUBSISTENCIA }\end{array}$ \\
\hline \multirow{2}{*}{$\begin{array}{l}\text { Regulación } \\
\text { por el Estado }\end{array}$} & Cumplimiento de normas & Inserción formal & Inserción marginal \\
\cline { 2 - 4 } & Incumplimiento de normas & Inserción sancionable & Inserción eventual \\
\hline
\end{tabular}

Fuente: Elaboración propia.

15 Más allá del registro de la actividad como una empresa, es posible una regulación mínima de parte del Estado aplicable a actividades de muy baja escala, normalmente un impuesto que no varía por la renta y que pecha el funcionamiento de la actividad, no se necesita siquiera que el negocio esté registrado como una empresa. En el encuestamiento de 2007 y 2010 se observó cómo esta regulación es frecuente en comercios minoristas que funcionan en mercados cuyo dueño es el municipio. Es común que el reconocimiento del Estado se traduzca en algún beneficio para el comerciante, por lo que este hace lo necesario para conseguirlo. En los mercados municipales el pago de un impuesto permite usufructuar un local comercial construido por el gobierno.

16 Piense en un vendedor ambulante de café que en la mañana se detiene en la acera con algunos termos y vocifera para ofrecer su producto. Para ejercer esta actividad solamente se debe estar atento al precio del café preparado en la lunchería o panadería cercanas para así no ofrecerlo más caro.

17 También llamada economía subterránea o sumergida. 
Así como la formalidad tiene regulaciones, la informalidad tiene sus regularidades ${ }^{18}$. Al menos, se escoge una actividad para trabajar, se define una cobertura geográfica de oferta del servicio y el tiempo de dedicación al trabajo, lo que establece regularidades en el intercambio que van haciendo un libre ajuste entre oferta y demanda (mediante una dinámica similar al establecimiento de costumbres o rutinas seguidas por muchas personas). Estas transacciones no están sujetas a la observancia de alguna regla dictada por alguien; sin embargo, la mayoría de las veces el intercambio se hace con prudencia para mantener en el tiempo la relación comercial. Un ejemplo es el taxista que trabaja de manera particular; sin la tarifa establecida por una compañía o por un taxímetro, este negociará su tarifa cada vez que un usuario solicite su servicio, considerando la distancia a recorrer, la hora del día, el precio que otros taxistas cobran, etc.; otros en iguales condiciones harán lo mismo. De una u otra manera se establece una regularidad en la tarifa del servicio de taxi no formal de una ciudad.

Tal como se presenta en el modelo de inserción, la informalidad no es homogénea; sin embargo, esta condición no se traduce en un asunto que rehúye al orden. Al contrario, una bondad del modelo es que ordena la inserción en cuatro contextos organizacionales, uno formal y tres informales. Estas distinciones podrían ayudar al estudio de las transacciones hechas fuera del aparato económico formal, además de aquellas que se dan entre cada tipo de inserción.

En las inserciones sancionable y marginal es más fácil evidenciar los intercambios con la economía formal. Los mismos se mantendrán siempre y cuando la normativa del Estado y las condiciones del mercado no sean tan estrictas como para impedirlos. A medida que la regulación amplía su espectro de acción sobre los intercambios, las actividades con inserción sancionable y marginal han de buscar alternativas para mantener sus transacciones, alguna de ellas podrán formalizarse (dependiendo de las condiciones en que esto ha de darse) o aumentar el rango de estrategias para que los intercambios se mantengan en el borde de la formalidad. Esto último es el efecto opuesto al esperado cuando el Estado toma la iniciativa de aumentar la fiscalización o las asociaciones formales de los agentes económicos (sindicatos, gremios de profesionales; cámaras de empresarios y sus respectivas federaciones; las bolsas de comercio, etc.) establecen normativas para el intercambio de bienes y servicios. En algunos contextos de inserción quienes realizan una actividad comercial de manera paralela a la formalidad, obtienen cierto tipo de ventaja respecto de quienes cumplen de manera estricta la legislación (por ejemplo, se pueden evitar las cargas impositivas); en otros contextos la informalidad limita el progreso de la actividad económica (por ejemplo, no poder negociar a cierta escala por la falta de documentación legal).

18 Existe un matiz entre la regulación proveniente del Estado y la proveniente del mercado. La regulación del Estado se dicta por normas siempre formales, por esto, en ocasiones hay asuntos no regulados; mientras que en el mercado la regulación puede ser formal o informal. Ejemplo de formalidad es cuando la transacción comercial está normada por reglas dictadas por alguien (gremio, contrato o similar); ejemplo de informalidad es cuando la transacción comercial se da por el libre ajuste entre quienes ofertan y quienes demandan un bien o servicio (es decir, sin que exista una bolsa comercial de por medio), aquí la norma no es dictada por alguien, sino más bien por las regularidades que se establecen al repetir muchas veces la misma transacción. Por tanto, en el mercado siempre habrá reglas que cumplir. 
En la inserción eventual, más que en el resto de la informalidad, la actividad puede desaparecer o ser reemplazada con facilidad, sin que quede afectado el funcionamiento de la economía dominante. Asimismo, la inserción es inestable y de poca duración, ya que carece de vínculos organizacionales (como aquellos vendedores ambulantes que hoy venden golosinas y mañana pintarán con brocha gorda alguna casa). Los inconvenientes y los costos por abandonar la actividad son menores, lo cual responde a que los recursos invertidos son mínimos (en algunos casos porque no se cuenta con estos). Esta inserción sirve para que la persona salga a ganarse la vida, pero no organiza una actividad con sentido económico. Se trata por tanto de un intercambio de mera subsistencia, es decir, dado a solamente conservar y mantener las transacciones sin estar orientado por un progreso de los vínculos organizacionales (es difícil Ilamar esto economía). Véase que en el modelo presentado en la Tabla 3, esta inserción no es paralela ni bordea la economía formal. En un contexto tal, el espíritu comercial consiste, básicamente, en la apropiación oportunista de la renta y no tanto en la planificación rentable, propia del empresario (Portes, 2004).

En contraposición, la inserción formal evidencia la existencia de un sistema organizacional. Demuestra el funcionamiento de una organización explícita en la economía local. La formalidad favorece la perdurabilidad de la actividad al orientarla a la inversión en capital fijo; se busca anclar la actividad, lo que genera costo al abandonarla. La formalidad es adecuada para una actividad económica organizada, económica por una racionalidad de gestionar recursos escasos (control de ingresos y egresos) y organizada al regirse por un contexto de regulaciones explícitas. El funcionamiento conjunto de varias actividades en este tipo de inserción crea un nicho económico estable y ofrece condiciones para un tejido organizacional robusto ${ }^{19}$.

Adviértase que la inserción organizacional eventual (u otra informal) no es sinónimo de pobreza, es decir, no contar con los bienes necesarios para cierto estándar de calidad de vida del hogar (sea ingresos, vivienda, salud, educación, etc.), por tanto -en esta inserción-, el hogar puede satisfacer sin mayor apremio sus necesidades de consumo. Tampoco trata

19 La noción de nicho es relevante para el estudio del tejido organizacional de la economía local. Téngase presente que toda actividad organizada (sea o no sea de carácter mercantil) "se coloca en redes de transacciones y procura acondicionar entre ellas un nicho en el que se estabilicen relaciones. El nicho - llamémoslo ecosocial- es un (micro) mundo de relaciones y credibilidades. Pero se cruzan allí regímenes generales de obligaciones que desde algún punto de vista son limitaciones y, desde otro, son seguridades y oportunidades" (Gruson, 2011: 15). Por ejemplo, una fábrica de carros es fuente de empleo para los habitantes de una ciudad, en esta empresa funciona un sindicato de obreros de la línea de ensamblaje y otro sindicato de los ingenieros del área de diseño, para ciertos intereses comunes ambos sindicatos se reúnen en uno solo, además, cada uno por separado es miembro de sus respectivas centrales sindicales; en un área de la fábrica funciona una guardería para los hijos de los trabajadores, por tanto, hay una sociedad de padres que está al tanto de la calidad de este servicio; por su parte, la fábrica de automóviles forma parte de una cámara empresarial de la ciudad y es una sucursal de su casa matriz que se encuentra en el extranjero, finalmente, esta empresa se vincula con una red de proveedores locales, nacionales e internacionales de bienes y servicios. Ciertamente, en una localidad no todas las organizaciones ejercerán una atracción tal como la empresa del ejemplo, sin embargo, esta ilustra bien cómo un entramado de transacciones estables forma la inserción organizacional de cualquier actividad. Aquí, la idea del nicho ecosocial es útil, a pesar de que en el texto que sirvió de inspiración el autor hace referencia al mismo desde una perspectiva sociopolítica. 
sobre la bondad del negocio en el sentido de las capacidades técnicas o profesionales del ocupado para ejercer la actividad organizada. Menos aún hace referencia a las expectativas o pretensiones individuales del dueño respecto de su negocio (hacerlo crecer o mantenerlo tal como está). La inserción trata del desarrollo organizacional en el que se desenvuelve la economía. Volviendo al caso del taxista que trabaja en la economía de subsistencia y que (pudiendo ser ingeniero) maneja su carro ocho horas diarias de lunes a viernes y el tiempo sobrante alquila el vehículo a otro taxista; en una ciudad grande el ingreso mensual que esto genera puede ser, en primer lugar, apreciable al estar libre de cualquier fiscalización $y$, en segundo lugar, mayor que el de otra persona cuya actividad está formalizada, sin embargo los vínculos con el sistema organizacional de la economía son nulos. Ciertamente, la informalidad puede ser disfuncional para la economía dominante, pero en cantidad de casos es funcional para actividades de muy pequeña escala.

\section{DESCRIPCIÓN DE LOS ENCUESTADOS: EJEMPLO DEL USO DE LA VARIABLE INSERCIÓN}

Para mostrar un primer uso de la variable Inserción organizacional se presenta a continuación una síntesis descriptiva de las microempresas consideradas en el encuestamiento de 2007 y 2010. Se comienza con la distribución según la rama de actividad en la que se clasifica el negocio. Adviértase que, en ocasiones, identificar negocios de escala micro con una rama de actividad sobredimensiona las tareas y transacciones realizadas; por ejemplo, si bien la categoría "Fabricación de alimentos" clasifica a una fábrica de panes que vende al mayor, también clasifica el empaquetado de semillas (maní, girasol, etc.) realizado en casa para ser vendido de manera ambulante.

Hay actividades que por su dinámica no pueden insertarse de cualquier manera en la colectividad, por ejemplo, no hay comercios mayoristas funcionando en una inserción eventual: el volumen de sus transacciones y las relaciones que deben establecer con sus proveedores no permiten obviar los controles formales. Al contrario, actividades agrícolas y pesqueras de escala micro son propensas a una inserción socioeconómica prácticamente nula, sobre todo por encontrarse en poblados rurales donde la fiscalización del Estado y las regularidades formales del mercado son escasas.

La construcción civil de escala microempresarial no siempre es una actividad precaria (inserción eventual); aunque en el encuestamiento la mayoría resultan ser albañiles de baja calificación autoempleados, casi la mitad hacen transacciones comerciales formales o al borde de la formalidad (inserción sancionable). Al contrario, los Servicios médicos se inscriben (casi) exclusivamente dentro de un contexto formal, la fiscalización en el ejercicio de las profesiones vinculadas a estos servicios dan poco espacio a la informalidad. Finalmente, si bien en las modalidades de inserción extrema se encuentra el mayor número de casos (la total formalidad $47 \%$ y la total informalidad 30\%), la zona intermedia (el 23\% restante) evidencia la existencia de las inserciones sancionable y marginal entre las microempresas del encuestamiento. 
En la siguiente Tabla se presentan algunos componentes usados para describir una empresa. La finalidad es mostrar que el componente (por ejemplo, el número de ocupados) se manifiesta de forma diferente al distinguir tres tipos de informalidad, constatando que la variable ayuda a observar con mayor detalle la segmentación que existe en la economía.

\section{TABLA 4}

Ramas de actividad en las que se encuestaron microempresas, según su tipo de inserción, 2007 y 2010 (valores absolutos)

\begin{tabular}{|c|c|c|c|c|c|}
\hline \multirow{2}{*}{ RAMA DE ACTIVIDAD } & \multicolumn{4}{|c|}{ INSERCIÓN ORGANIZACIONAL } & \multirow{2}{*}{ TOTALES } \\
\hline & FORMAL & SANCIONABLE & MARGINAL & EVENTUAL & \\
\hline Agricultura y pesca & 3 & 3 & 3 & 23 & 32 \\
\hline Fabricación de alimentos & 16 & 6 & 6 & 37 & 65 \\
\hline Fabricación textil-calzado & 30 & 9 & 8 & 22 & 69 \\
\hline Fabricación con madera & 22 & 10 & 5 & 16 & 53 \\
\hline Fabricación con papel & 28 & 3 & 0 & 2 & 33 \\
\hline Fabricación con metal & 22 & 4 & 5 & 26 & 57 \\
\hline Construcción civil & 12 & 10 & 1 & 30 & 53 \\
\hline Comercio mayorista & 28 & 1 & 1 & 0 & 30 \\
\hline Comercio minorista & 131 & 49 & 49 & 47 & 276 \\
\hline Comida y bebida preparada & 48 & 10 & 15 & 28 & 101 \\
\hline Transporte urbano & 52 & 23 & 7 & 37 & 119 \\
\hline Servicios a empresas & 37 & 11 & 0 & 16 & 64 \\
\hline Servicios médicos & 26 & 4 & 0 & 3 & 33 \\
\hline Servicios de reparación & 49 & 7 & 3 & 27 & 86 \\
\hline Servicios personales & 44 & 11 & 10 & 30 & 95 \\
\hline Totales & 548 & 161 & 113 & 344 & 1.166 \\
\hline$\%$ & 47 & 14 & 9 & 30 & 100 \\
\hline
\end{tabular}

Fuente: Bases de datos de microempresas 2007 y 2010, elaboradas en CISOR. 
El número de ocupados no es independiente del tipo de inserción de la actividad económica. El tamaño del negocio encuentra su límite más pronto en la informalidad, sobre todo cuando la inserción es eventual. Véase en la Tabla 5 que son pocas las empresas informales con 5 a 10 ocupados. Asimismo, aunque la informalidad gira en torno al micronegocio, los mismos son igualmente funcionales en condiciones formales.

Por otro lado, una distribución de los dueños de negocios según su nivel de instrucción es diferenciable por los contextos organizacionales en los que se inserta la actividad económica. Los rangos de escolaridad extremos (hasta 6 años y 12 o más años) ejemplifican bien esta distinción. Cuando se ha estudiado hasta 6to grado, de la inserción formal a la eventual la proporción de dueños aumenta; al contrario, cuando se ha estudiado 12 o más años (se es bachiller o se tiene estudios superiores) de la inserción formal a la eventual la proporción de dueños decrece.

\section{TABLA 5}

Tamaño del negocio y nivel de instrucción del dueño diferenciados por tipo de inserción, encuestamientos de 2007 y 2010 (valores porcentuales)

\begin{tabular}{|c|c|c|c|c|c|}
\hline \multirow[b]{2}{*}{ VARIABLE A DESCRIBIR } & \multicolumn{4}{|c|}{ INSERCIÓN ORGANIZACIONAL } & \multirow[b]{2}{*}{ TOTAL } \\
\hline & FORMAL & SANCIONABLE & MARGINAL & EVENTUAL & \\
\hline \multicolumn{6}{|l|}{ \# de ocupados en el negocio } \\
\hline Unipersonal & 20 & 46 & 37 & 60 & 37 \\
\hline 2 a 4 & 48 & 44 & 54 & 40 & 46 \\
\hline 5 a 10 & 26 & 10 & 9 & 0 & 15 \\
\hline 11 y más & 6 & 0 & 0 & 0 & 3 \\
\hline \multicolumn{6}{|c|}{ Años de escolaridad del dueño } \\
\hline hasta 6 & 14 & 22 & 25 & 32 & 21 \\
\hline 7 a 9 & 11 & 19 & 19 & 26 & 17 \\
\hline 10 a 11 & 36 & 35 & 42 & 29 & 35 \\
\hline 12 o más & 39 & 24 & 14 & 13 & 27 \\
\hline Totales & 100 & 100 & 100 & 100 & 100 \\
\hline
\end{tabular}

Fuente: Bases de datos de microempresas 2007 y 2010, elaboradas en CISOR. 
En relación con los asuntos administrativos y gerenciales vinculados a la regulación de la actividad, a continuación se describe el cumplimiento de las obligaciones laborales, los aspectos contables y la remuneración del dueño.

TABLA 6

Cumplimiento de formalidades administrativas diferenciadas por tipo de inserción, encuestamientos de 2007 y 2010 (valores porcentuales)

\begin{tabular}{|l|l|c|c|c|c|c|}
\hline \multirow{2}{*}{ VARIABLE A DESCRIBIR } & \multicolumn{4}{|c|}{ INSERCIÓN ORGANIZACIONAL } & \multirow{2}{*}{ TOTAL } \\
\cline { 3 - 7 } & FORMAL & SANCIONABLE & MARGINAL & EVENTUAL & \\
\hline $\begin{array}{l}\text { Obligaciones } \\
\text { laborales* }\end{array}$ & Sí & 39 & 0 & 0 & 0 & 19 \\
\cline { 2 - 7 } & No & 61 & 100 & 100 & 100 & 81 \\
\hline $\begin{array}{l}\text { Contabilidad } \\
\text { del negocio }\end{array}$ & Sí & 75 & 15 & 0 & 0 & 40 \\
\cline { 2 - 7 } & No & 25 & 85 & 100 & 100 & 60 \\
\hline $\begin{array}{l}\text { Remuneración } \\
\text { del dueño** }\end{array}$ & Regular & 61 & 52 & 56 & 0 & 41 \\
\cline { 2 - 7 } & Irregular & 39 & 48 & 44 & 100 & 59 \\
\hline & 100 & 100 & 100 & 100 & 100 \\
\hline
\end{tabular}

Fuente: Bases de datos de microempresas 2007 y 2010, elaboradas por CISOR.

* Las obligaciones establecidas en la ley en Venezuela son: seguridad social (atención en salud y fondo de pensión), ahorro para la vivienda y paro forzoso.

** La remuneración es regular cuando el dueño recibe un salario o sueldo; e irregular cuando el dueño toma lo que necesita del dinero del negocio.

Los datos de la Tabla 6 ilustran que el cumplimiento de obligaciones laborales es un aspecto propio de la inserción formal (39\%), sin embargo, es evidente que este contexto está matizado por lo estricto que puedan ser los entes fiscalizadores con los negocios de escala microempresarial. Véase que el $61 \%$ de los encuestados de inserción formal no cumple con las obligaciones laborales. Así, la formalidad no se traduce siempre en condiciones de contratación robusta o no precarias ${ }^{20}$. Ocurre de igual manera con la contabilidad, en la

20 Un estudio que toca el tema de la estabilidad y precariedad del empleo, pero en el contexto de los habitantes de algunos barrios de Caracas, es: Cecilia Cariola (Coord.) (1992), Sobrevivir en la pobreza: el fin de una ilusión. Caracas: CENDES / Nueva Sociedad. Citado en Gruson (2006). En la página 18, este último indica que (para quienes trabajan en los barrios estudiados por Cariola) si bien "el empleo informal es más a menudo precario que el empleo formal, las diferencias proporcionales no son tantas como para asimilar lo formal con la estabilidad, lo informal con lo precario". Disponible en: www.cisor.org.ve 
formalidad algunas empresas encuentran un espacio de flexibilidad y funcionan sin hacer registros contables $(25 \%)$; a pesar de que gracias a la contabilidad se conoce el vaivén de los estados financieros y se pueden prevenir percances para el negocio. El no cumplimiento de ambos requisitos administrativos en la inserción marginal y eventual evidencia que la necesidad de practicarlos es generada por la inserción en que se encuentra la actividad, y no por la iniciativa del empresario. Sin embargo, siempre habrá quienes hacen algún tipo de anotación del flujo de dinero de su negocio, sobre todo cuando las cantidades transadas lo ameritan, pero estos datos (por mejor elaborado que estén) no son una contabilidad formal declarable.

Llama la atención la contabilidad declarada por quienes se insertan en una actividad organizacional sancionable. En este caso, puede ser que la actividad, estando registrada, no declare al fisco. Sin embargo, por hacer transacciones en la economía dominante (donde el indicador es el respaldo de las facturas), en la organización se elaboran los estados contables de manera regular.

La forma de remunerar el trabajo del dueño no responde a un tipo de inserción, con excepción de la eventual donde siempre es irregular. Por tanto, en empresas con inserción formal o que bordean la formalidad, se dan condiciones internas que favorecen o no la regularidad de la remuneración. Por ejemplo, si el flujo de capital es reducido, establecer una remuneración fija puede resultar un compromiso difícil de cumplir. La forma de remuneración parece ser, en casos como este, indicador del rango de libertad del dueño para gerenciar su negocio ${ }^{21}$.

\section{CONCLUSIONES}

El esfuerzo ha sido presentar una variable compuesta de manera empírica que ilustre un concepto sociológico de inserción, indicando la temática en que se enmarca y evidenciando la metodología para su elaboración. Así, se identificaron los componentes elementales que diferencian contextos de inserción organizacional. Si bien estos contextos han sido elaborados a partir de transacciones, los cuatro tipos de inserción no son formas de intercambio, más bien, son una estructura normativa que influye en los procesos de intercambio, mientras que estos últimos son relaciones de supraordinación o subordinación, competencia o cooperación, y las mutuas dependencias; siendo todas estas relaciones (en mayor frecuencia) desiguales ${ }^{22}$. Por otro lado, estos intercambios se dan a lo interno de cada contexto o de un contexto a otro; por ejemplo, un grupo de costureras que trabajan -cada una- en su casa venden la ropa que elaboran a una fábrica textil que cumple todas las normas de formalidad con el

21 Se puede pensar que parte del desarrollo de la microempresa implica un control de la remuneración del dueño, si bien esto puede ser cierto desde un punto de vista del dominio o control interno de la organización, ya que es la consecuencia de separar las finanzas familiares y personales de las del negocio; desde el punto de vista de los contextos más amplios de intercambio, este control de la remuneración no está vinculado a un funcionamiento formal de la actividad económica.

22 Puede verse una alusión al tema en el trabajo de Gruson, 2006, pp. 21, en el que se hace una referencia al trabajo de Consuelo Iranzo y Jacqueline Richter (2005), La subcontratación laboral. Bomba de tiempo contra la paz social. Caracas: UCV, 174 p. Gruson señala que el capítulo 4 (pp. 81-134) considera el caso de Venezuela. 
Estado (registro legal y pago de impuesto) y con el mercado (entrega de factura legal a su proveedor); en este caso el intercambio (posiblemente de subordinación de las costureras para con la fábrica textil) se da entre un contexto de inserción sancionable y uno formal.

Una definición de inserción desde como la autonomía de la organización y su subordinación al orden social definen los nexos entre las actividades en la economía, orienta el análisis hacia distinguir la existencia de un sistema organizacional formal, que termina siendo un bien civilizatorio, más allá de aquellas transacciones que funcionan solamente para la mera subsistencia de los involucrados inmediatos. Una vez hecha estas distinciones, se puede planear el desarrollo (también) a partir del progreso de los vínculos organizacionales en la economía.

Los cuatro órdenes explicitan condiciones que establecen más o menos regularidad y previsibilidad en las relaciones. En la formalidad hay obligaciones legales que se deben cumplir, aquí el devenir de la transacción está explicitado antes que esta inicie, lo que permite establecer controles administrativos en los intercambios de bienes y servicios. En la (total) informalidad solo hay ciertas regularidades que se deben asumir, aquí el devenir de la transacción -en todo caso- se explicita únicamente mediante repetidas transacciones. Estas condiciones significan diferencia en la previsibilidad de los resultados que se pueden obtener en los intercambios (saber a qué atenerse al momento de iniciarlo), involucrando distintas condiciones y requisitos, riesgos y seguridades, compromisos y confianzas. Así, cada tipo de inserción conforma un régimen para la actividad que se ejerce y es un segmento de una economía de racionalidad heterogénea. Desde este punto de vista, en la formalidad se está en la economía moderna por el hecho fundamental de establecer previsibilidad (dominio) en los procesos propios de un intercambio económico. Siendo que la inserción es una relación entre organizaciones, entonces, tanto formalidad como informalidad no hace referencia directa a la persona (el ocupado), sino al contexto para el intercambio. Así, el individuo tiene trayectorias personales en su vida profesional, pero son las organizaciones las que componen una estructura necesaria para las transacciones generalizadas que forman la economía. El planteamiento es llevar la discusión de la inserción de actividades socioproductivas al plano organizacional y no dejarlo en el plano de la persona (al referirse, por ejemplo, a su nivel educativo) o de una característica administrativa de la empresa (al referirse, por ejemplo, a su número de ocupados).

Entonces, formalidad e informalidad se plantean como la variante de una cualidad inter-organizacional, y no como una cualidad particular de la empresa, de la ocupación o de los ocupados; sin restar importancia a estos tres puntos de vista que forman parte importante del estudio de la dinámica del trabajo. Ciertamente, no se pretende olvidar que son las personas quienes se desenvuelven en una actividad económica más o menos organizada, sin embargo, la inserción de la actividad en la economía es un hecho de índole organizacional. La idea es de contextos formales y contextos informales, lo que permite explicitar una estructura para los intercambios que son propios de las organizaciones. Así, formalidad e informalidad son condiciones de inserción de la organización en la actividad económica y no de la persona. 
El modelo de inserción ha sido elaborado a partir de datos proporcionados por negocios de pequeña y muy pequeña escala, elección pertinente porque estas actividades económicas permitieron distinguir la variedad de inserciones informales propiamente locales, principalmente la eventual, ya que ninguna empresa de cierta dimensión puede funcionar con una inserción organizacional prácticamente nula en la economía. Si bien el modelo ha sido elaborado y ejemplificado desde las actividades comerciales de escala micro, este parece ser válido para cualquier actividad organizada, con o sin fin de lucro, del sector privado o público (siendo que las actividades del Estado siempre deberían ser formales).

\section{BIBLIOGRAFÍA}

Cacciamali, Maria Cristina (2007): "(Pre-) Conceito sobre o setor informal, reflexões parciais embora instigantes", Econômica, 9 (1), pp. 145-168.

Centro de Investigación Social (2005): La microempresa en Venezuela. Caracterización por medio de la encuesta de hogares. Estudio piloto sobre los años 1995 y 2000, CISOR, Caracas.

Chantal, Jean-François y Francine Seguin (1987): L'analyse des organisations, une anthologie sociologique. Tome 2: les composantes de l'organisation, Gaëtan Morin Editeur, Québec.

Diccionario de la Real Academia Española, en http://www.rae.es

Ejecutivo Nacional: "Requisitos para el registro de una empresa", en http://www.gobiernoenlinea.gob.ve

Feijo, Carmem, Denise Do Nascimento y Augusto De Souza (2009): "Quão heterogêneo é o setor informal brasileiro? Uma proposta de classificação de atividades baseada na Ecinf", Revista de Economia Contemporânea, 13 (2), pp. 329-354.

García, Jenny, Carlos Santos y Laura Tovar (2009): Posiciones geosociales: estratos sociales y ámbitos urbano-regionales. Venezuela 1985-2005, ponencia presentada en el "IV Encuentro Nacional de Demógrafos y Estudiosos de la Población", 13-15 de mayo, UCAB-Guayana, Puerto Ordaz.

Gruson, Alberto (2006): Preámbulo a un estudio de vulnerabilidad social. Centro de Investigación Social, CISOR, Caracas, en www.cisor.org.ve

(2008): "Un mapa de posiciones geosociales: estratos sociales y ámbitos urbano-regionales en Venezuela", Temas de Coyuntura, n58, Instituto de Investigaciones Económicas y Sociales, $\mathrm{UCAB}$, Caracas

(2011): El polo asociativo y la sociedad. Estudios para el caso venezolano, Sinergia, Caracas.

Instituto Nacional de Estadística (INE) (2006): Diccionario único comparativo de la Encuesta de Hogares por Muestreo, 1994-2005, INE, Caracas.

Oficina Central de Estadísticas e Información (OCEI) (1995): Diccionario normalizado de datos encuesta de hogares 1975 a 1994, OCEI, Caracas.

(1997): Encuesta de Hogares por Muestreo 1967-1997, OCEl, Caracas.

OIT (1992): Fuentes y métodos: estadísticas del trabajo. Volumen 3: población económicamente activa, empleo, desempleo y horas de trabajo (encuestas de hogares), OIT.

(2002): El trabajo decente y la economía informal, Conferencia Internacional del Trabajo, 90na Reunión, Génova. 
Portes, Alejandro y William Haller (2004): "La economía informal", Serie Políticas Sociales, División de Desarrollo Social, CEPAL, Santiago.

Ritzer, George (2002): "La teoría del intercambio de Peter Blau", en George Ritzer: Teoría sociológica moderna, McGraw-Hill, Madrid, pp. 349-355.

Touraine, Alain (1993 [1973]): Production de la société, Le Livre de Poche, Paris.

Zambrano, Andrés (2004): Economía e informalidad", Reporte Venescopio, 2, CISOR, Caracas, en http://www.venescopio.org.ve

(2007): El micro-seguro en Venezuela. Una exploración en el sector micro-empresarial, CISOR, Caracas.

Zambrano, Andrés y Henry Moncrieff (2011): AUGE, el programa de Apoyo a Unidades de Gestión Económica del Centro al Servicio de la Acción Popular CESAP. Tras 15 años de experiencia. Venezuela 1995-2010, CISOR, Caracas.

Recibido: 28-01-2013

Aceptado: 17-06-2013 